\title{
Prophylaxie pour la pneumonie à Pneumocystis jirovecii chez une femme de 42 ans suivant un traitement immunosuppresseur
}

\author{
Sheliza Halani MD, Nisha Andany MD MSP, Rupal Shah MD MEPS
}

citation : CMAJ 2020 October 26;192:E1206-8. doi : 10.1503/cmaj.200846-f

Voir la version anglaise de l'article ici : www.cmaj.ca/lookup/doi/10.1503/cmaj.200846

$\mathbf{U}$

ne femme de 42 ans atteinte depuis 2 ans de dermatomyosite contrôlée avec de la prednisone $(5 \mathrm{mg}, 1$ fois par jour) se présente chez son médecin de famille une semaine après une poussée de la maladie qui a nécessité une hospitalisation. La patiente présente une faiblesse des muscles proximaux et une dyspnée causée par une maladie pulmonaire interstitielle. Le traitement amorcé est une dose élevée de prednisone (60 $\mathrm{mg}$ par jour, par voie orale), qui sera réduite très graduellement. Le médecin de famille se demande s'il devrait prescrire une prophylaxie à la patiente pour prévenir une pneumonie à Pneumocystis jirovecii.

\section{La patiente est-elle susceptible de contracter une pneumonie à Pneumocystis jirovecii?}

La pneumonie à Pneumocystis jirovecii est une infection fongique opportuniste ${ }^{1,2}$. Le déficit de l'immunité cellulaire est le principal facteur de risque de cette maladie, un lien qui a été particulièrement bien établi ${ }^{3}$ chez les patients atteints du VIH ayant une numération des CD4 inférieure à 200/mm³. Pourtant, l'incidence croissante de la pneumonie à Pneumocystis jirovecii chez les personnes n'ayant pas le VIH (encadré 1) met en évidence le rôle de l'immunosuppression indépendamment du VIH ${ }^{1-5}$. De plus, les glucocorticoïdes affaiblissent l'immunité cellulaire en réduisant le compte de lymphocytes CD4+ et en limitant la cascade de signalisation inflammatoire ${ }^{3,6}$. Bien que le risque soit le plus élevé pour les personnes prenant des corticostéroïdes et atteintes d'une affection sous-jacente qui augmente le risque de pneumonie à Pneumocystis jirovecii (hémopathie maligne) ou prenant d'autres agents immunosuppresseurs, la monothérapie a aussi été associée à cette maladie ${ }^{3}$. Dans une étude prospective de cohorte portant sur des patients atteints de troubles rhumatologiques qui prenaient de la prednisone $(\geq 30 \mathrm{mg}$ par jour pendant au moins 4 semaines), l'incidence de la pneumonie à Pneumocystis jirovecii à 1 an était de 2,37 cas par 100 personnes-années ${ }^{7}$. Les groupes prenant une dose plus élevée de prednisone ( $\geq 60 \mathrm{mg}$ ), utilisant une thérapie pulsée à la cyclophosphamide et ayant un âge plus avancé ou une lymphocytopénie au début de l'étude étaient plus à risque $^{7}$. Une analyse rétrospective s'intéressant à des patients n'ayant pas le VIH a révélé que la pneumonie à Pneumocystis jirovecii pouvait se développer avec des doses de corticostéroïdes aussi faibles que $16 \mathrm{mg} /$ jour prises pendant 8 semaines ou moins ${ }^{3}$. Les antécédents de dermatomyosite de la patiente et la récente poussée exigeant une forte dose de prednisone augmentent son risque de contracter une pneumonie à Pneumocystis jirovecii.

\section{Comment la pneumonie à Pneumocystis jirovecii se présenterait-elle chez cette patiente?}

Le présentation clinique de ce type de pneumonie varie et est non spécifique, mais chez cette patiente, les symptômes typiques incluraient une dyspnée aiguë, de la toux et de la fièvre pendant plusieurs jours ${ }^{8}$, ce qui ne concorde pas avec l'apparition indolente des symptômes se produisant sur plusieurs jours ou semaines observée chez les patients atteints du $\mathrm{VIH}^{8}$. Une étude prospective réalisée en France en 2014 a conclu que les patients atteints d'une pneumonie à Pneumocystis jirovecii qui n'avaient pas le VIH présentaient moins de symptômes à l'apparition de la maladie (ce qui retarde le diagnostic), commençaient le traitement plus tard et avaient un plus haut taux de ventilation mécanique et de décès que les patients atteints du $\mathrm{VIH}^{8}$.

Les cliniciens devraient maintenir un fort degré de suspicion quant à la pneumonie à Pneumocystis jirovecii chez les patients immunosupprimés non atteints du VIH, puisqu'il peut être difficile de confirmer le diagnostic. Par ailleurs, les marqueurs de substitution ne sont pas utiles; les tests sur le taux de lactate-déshydrogénase ont une sensibilité de $90 \%$ pour les patients atteints du VIH, mais de $63 \%$ seulement pour les autres patients ${ }^{9}$. Une tomodensitométrie thoracique montrera des opacités bilatérales en verre dépoli dans les 2 populations, mais la confirmation du diagnostic exige une visualisation directe du pathogène en cause ${ }^{4}$. Les méthodes de détection comme le lavage bronchoalvéolaire ont une sensibilité moins importante chez les patients qui n'ont pas le VIH parce que la quantité de micro-organismes chez eux est moins importante ${ }^{4}$. 
Encadré 1 : Indications pour la prophylaxie de la pneumonie à Pneumocystis jirovecii chez les patients immunodéprimés et séronégatifs au VIH

\section{Populations de}

patients

\section{Indications pour la prophylaxie}

Transplantation d'organes pleins

Greffe de cellules souches

hématopoïétiques

Tumeur solide

Maladie auto-immune et vascularite

Déficit immunitaire primaire

Traitement par des glucocorticoïdes (autre)†

Pendant 6-12 mois après la transplantation*

Greffe de cellules souches autologues

- Pendant 3-6 mois

Allogreffe de cellules souches

- Pendant au moins 6 mois

- Une période plus longue peut être nécessaire en cas de réaction chronique du greffon contre l'hôte (GVH) ou de traitement immunosuppresseur actif

Glucocorticoïdest équivalant à une dose de prednisone $\geq 20 \mathrm{mg} /$ jour pendant 4 semaines

Leucémie lymphoblastique aiguë traitée par chimiothérapie

Alemtuzumab

- Pendant le traitement et au moins 2 mois après la fin (et jusqu'à ce que la numération des $C D 4$ soit supérieure à 200 cellules $/ \mathrm{mm}^{3}$ )

Agents réduisant la quantité de lymphocytes $T$ (p. ex., fludarabine ou cladribine)

- Jusqu'à ce que la numération des CD4 soit supérieure à 200 cellules $/ \mathrm{mm}^{3}$

Glucocorticoïdest équivalant à une dose de prednisone $\geq 20 \mathrm{mg} /$ jour pendant $\geq 4$ semaines

Témozolomide et radiothérapie

- Jusqu'à ce que la numération des CD4 soit supérieure à 200 cellules $/ \mathrm{mm}^{3}$

Arthrite rhumatoïde traitée par des inhibiteurs du facteur de nécrose tumorale a

- Si des glucocorticoïdes† ou d'autres agents immunosuppresseurs sont aussi administrés

Maladie inflammatoire de l'intestin traitée par des inhibiteurs du

facteur de nécrose tumorale $\alpha$

- Si des glucocorticoïdes $†$ ou d'autres agents immunosuppresseurs sont aussi administrés

Granulomatose avec polyangéite traitée par de la cyclophosphamide

Vascularite associée à des ANCA

- Traitée par de la cyclophosphamide et des glucocorticoïdes†

Autre vascularite

- Traitée par des glucocorticoïdes† et un deuxième agent immunosuppresseur

Maladie des tissus conjonctifs

- Traitée par des glucocorticoïdest équivalant à une dose de prednisone $\geq 20 \mathrm{mg} /$ jour pendant $\geq 4$ semaines

Déficit immunitaire combiné sévère

Lymphocytopénie CD4 idiopathique

Syndrome hyper-IgM lié à l'X

Équivalent d'une dose de prednisone $\geq 20 \mathrm{mg} /$ jour pendant

$\geq 4$ semaines, et au moins un des éléments suivants:

- Autres agents immunosuppresseurs

- Antirhumatismaux non stéroïdiens

- Trouble immunosuppresseur sous-jacent

\section{Autre population de patient (pour laquelle la prophylaxie peut être envisagée)}

Traitement par des glucocorticoïdes†
Équivalent d'une dose de prednisone $\geq 20 \mathrm{mg} /$ jour pendant $\geq 4$ semaines - Avec ou sans maladie pulmonaire sous-jacente

Remarque : $\mathrm{ANCA}=$ anticorps anticytoplasme des polynucléaires neutrophiles, $\operatorname{lgM}=$ immunoglobuline $\mathrm{M}$.

*La durée varie selon l'organe transplanté et les exigences relatives aux autres agents immunosuppresseurs (chez les patients transplantés rénaux, la prophylaxie pourrait être écourtée).

†Glucocorticoïdes (équivalant à une dose de prednisone $\geq 20 \mathrm{mg} /$ jour pendant $\geq 4$ semaines; certains experts recommandent $\geq 16-20 \mathrm{mg} /$ jour).

\section{La patiente devrait-elle recevoir une prophylaxie pour la pneumonie à Pneumocystis jirovecii?}

La décision d'amorcer une prophylaxie chez cette patiente exige une prise en compte soigneuse des risques et des avantages; elle est toutefois appuyée par la science ${ }^{7}$. Ce traitement est recommandé quand le risque de pneumonie à Pneumocystis jirovecii est supérieur à $3,5 \%{ }^{5}$. Les données appuyant la prophylaxie chez les patients n'ayant pas le $\mathrm{VIH}$ sont les plus solides pour la transplantation d'organes solides ou de cellules souches hématopoïétiques, pour les tumeurs solides ou les agents réduisant la quantité de lymphocytes $\mathrm{T}$, pour les hémopathies malignes, pour le déficit immunitaire primaire, pour les vascularites et pour les maladies auto-immunes traitées par des inhibiteurs du facteur de nécrose tumorale $\alpha$ (encadré 1$)^{1,2,4,5}$.

Dans 2 méta-analyses comparant la prophylaxie à triméthoprime-sulfaméthoxazole (TMP-SMX) et un placébo ou l'absence de prophylaxie chez des patients à risque atteints d'une hémopathie maligne ou ayant subi une transplantation, on a rapporté un nombre de sujets à traiter aussi faible que 15 et 19 pour prévenir 1 cas de pneumonie à Pneumocystis jirovecii; le taux global d'événements indésirables était de $3,1 \%$, sans différence entre les groupes ${ }^{1,5}$.

Chez les personnes atteintes de maladies des tissus conjonctifs, l'incidence de pneumonie à Pneumocystis jirovecii est la plus élevée en présence de granulomatose avec polyangéite ( $8 \%-12 \%)$, de polyartérite noueuse $(6,5 \%)$ et de dermatomyosite ou de polymyosite $(2,7 \%)^{4}$. Bien que le risque isolé de pneumonie à Pneumocystis jirovecii tombe sous 3,5\% pour les myopathies inflammatoires, ces troubles sont associés à une diminution des lymphocytes $T^{10}$ et exigent souvent un traitement par glucocorticoïdes; et une maladie pulmonaire interstitielle concomitante accroît le risque ${ }^{11}$. Dans une étude de cohorte portant sur 1092 patients atteints de troubles rhumatologiques qui prenaient une dose modérée à élevée de stéroïdes, certains patients ont reçu une prophylaxie à TMP-SMX selon le jugement du médecin traitant ${ }^{7}$. La prophylaxie a réduit l'incidence de la pneumonie à Pneumocystis jirovecii à un an de $93 \%$ et la 
mortalité associée de $92 \% \%^{7}$. Le nombre de sujets à traiter pour prévenir un cas de pneumonie à Pneumocystis jirovecii était de 52; les réactions pulmonaires négatives graves étaient rares (nombre requis pour causer un préjudice $=131)^{7}$. En l'absence de lignes directrices claires, la littérature favorise la prophylaxie chez cette patiente atteinte de dermatomyosite et de maladie pulmonaire interstitielle qui prend d'importantes doses de stéroïdes pour de longues périodes.

\section{Quelles sont les options de prophylaxie pour la pneumonie à Pneumocystis jirovecii?}

L'agent favorisé est le TMP-SMX². Il peut être administré de 2 manières : 1 comprimé à dose double 3 fois par semaine, ou 1 comprimé à dose normale chaque jour - il n'y a aucune différence d'efficacité ou de toxicité ${ }^{1}$. Les effets secondaires incluent les éruptions cutanées, la myélosuppression, l'hépatotoxicité et la néphrotoxicité ${ }^{1,7}$. Les autres options seraient la dapsone, l'atovaquone et la pentamidine en aérosol, selon les données limitées et largement extrapolées provenant de publications sur des personnes atteintes du $\mathrm{VIH}^{2,4}$.

\section{Retour à l'étude de cas}

La patiente présente de multiples facteurs de risque de la pneumonie à Pneumocystis jirovecii, notamment une maladie rhumatologique sous-jacente qui requiert d'importantes doses de glucocorticoïdes. Si la pneumonie se développe, la patiente est à risque d'une infection grave et de mauvais résultats pour la santé, surtout en raison de sa maladie pulmonaire interstitielle. Selon les données disponibles, les avantages de la prophylaxie surpassent les risques de préjudices. Après consultation en rhumatologie, la patiente a amorcé une prophylaxie à TMP-SMX, et il y a eu un suivi des réactions négatives.

\section{Références}

1. Stern A, Green H, Paul M, et al. Prophylaxis for Pneumocystis pneumonia (PCP) in non-HIV immunocompromised patients. Cochrane Database Syst Rev 2014;2014:CD005590.

2. Carmona EM, Limper AH. Update on diagnosis and treatment of Pneumocystis pneumonia. Ther Adv Respir Dis 2011;5:41-59.

3. Yale SH, Limper AH. Pneumocystis carinii pneumonia in patients without acquired immunodeficiency syndrome: associated illnesses and prior corticosteroid therapy. Mayo Clin Proc 1996;71:5-13.

4. Roux A, Gonzalez F, Roux M, et al. Groupe de recherche respiratoire en réanimation en onco-hématologie (Grrr-OH). Update on pulmonary Pneumocystis jirovecii infection in non-HIV patients. Med Mal Infect 2014;44:185-98.

5. Green H, Paul M, Vidal L, et al. Prophylaxis of Pneumocystis pneumonia in immunocompromised non-HIV-infected patients: systematic review and metaanalysis of randomized controlled trials. Mayo Clin Proc 2007;82:1052-9.

6. Cain DW, Cidlowski JA. Immune regulation by glucocorticoids. Nat Rev Immunol 2017;17:233-47.
7. Park JW, Curtis JR, Moon J, et al. Prophylactic effect of trimethoprimsulfamethoxazole for Pneumocystis pneumonia in patients with rheumatic diseases exposed to prolonged high-dose glucocorticoids. Ann Rheum Dis 2018;77:644-9.

8. Roux A, Canet E, Valade S, et al. Pneumocystis jirovecii pneumonia in patients with or without AIDS, France. Emerg Infect Dis 2014;20:1490-7.

9. Vogel M, Weissgerber P, Goeppert B, et al. Accuracy of serum LDH elevation for the diagnosis of Pneumocystis jirovecii pneumonia. Swiss Med Wkly 2011;141:w13184.

10. Zhang L, Xia Q, Li W, et al. The RIG-I pathway is involved in peripheral T-cell lymphopenia in patients with dermatomyositis. Arthritis Res Ther 2019;21:131.

11. Kadoya A, Okada J, likuni Y, et al. Risk factors for Pneumocystis carinii pneumonia in patients with polymyositis/dermatomyositis or systemic lupus erythematosus. J Rheumatol 1996;23:1186-8.

Intérêts concurrents : Nisha Andany déclare sa participation en tant qu'investigateur de site pour des essais cliniques sur le VIH financés par Gilead Sciences, GlaxoSmithKline et Janssen (externe au travail soumis). Aucun autre intérêt concurrent déclaré.

Cet article a été évalué par des pairs.

Il s'agit d'un scénario clinique fictif.

Affiliations : Faculté de médecine (Halani, Andany, Shah), Université de Toronto; division des maladies infectieuses (Andany), Centre des sciences de la santé Sunnybrook; division de médecine interne générale (Shah), Hôpital Western Toronto, Toronto (Ontario).

Collaborateurs : Tous les auteurs ont contribué à la conception du travail, ont rédigé le manuscrit et en ont révisé de façon critique le contenu intellectuel important; ils ont donné leur approbation finale pour la version destinée à être publiée et assument l'entière responsabilité de tous les aspects du travail.

Propriété intellectuelle du contenu : Il s'agit d'un article en libre accès distribué conformément aux modalités de la licence Creative Commons Attribution (CC BY-NC-ND 4.0), qui permet l'utilisation, la diffusion et la reproduction dans tout médium à la condition que la publication originale soit adéquatement citée, que l'utilisation se fasse à des fins non commerciales (c.-à-d., recherche ou éducation) et qu'aucune modification ni adaptation n'y soit apportée. Voir : https://creativecommons.org/licenses/by-nc-nd/4.0/.

Correspondance : Rupal Shah, rupal.shah@uhn.ca

Décisions est une série qui traite des approches pratiques fondées sur des données probantes pour des pathologies courantes en soins primaires. Les articles portent sur les décisions clés qu'un médecin peut devoir prendre lors d'un examen initial. La situation présentée doit habituellement pouvoir s'observer lors d'une consultation typique en soins primaires. Les articles ne doivent pas dépasser 650 mots, peuvent inclure un encadré, une figure ou un tableau et doivent commencer par une très brève description (75 mots ou moins) du cas clinique. Les décisions doivent être présentées sous forme de questions. On encourage la présentation d'un encadré renfermant quelques ressources utiles pour les patients ou les médecins. 